\title{
Quiste Epitelial Esplénico Gigante, Características Morfológicas y Evolución Postoperatoria. Reporte de un Caso
}

\author{
Epithelial Splenic Cyst. Morphological Characteristics and Postoperative Evolution. Case Report
}

\author{
Carlos Manterola ${ }^{1,2}$
}

MANTEROLA, C. Quiste epitelial esplénico gigante. Características morfológicas y evolución postoperatoria. Reporte de un caso. Int. J. Morphol., 39(2):667-671, 2021.

RESUMEN: Los quistes primarios del bazo (QPB), son lesiones poco frecuentes en patología quirúrgica; los mayores de $5 \mathrm{~cm}$ o sintomáticos deben ser tratados quirúrgicamente para evitar el riesgo de complicaciones. Se debe realizar un examen histopatológico para confirmar el subtipo de quiste esplénico y descartar una eventual transformación maligna del revestimiento epitelial pluripotencial. El objetivo de este manuscrito fue reportar un caso de QPB intervenido quirúrgicamente y revisar la evidencia existente respecto de sus características morfológicas, terapéuticas y pronósticas. Caso clínico: Se trata de una mujer de 18 años (MAC), que consultó por distensión abdominal progresiva, de varios meses de evolución. La tomografía abdominal reveló la existencia de una masa heterogénea de 21 cm de diámetro mayor, en relación con el colon transverso y la curva mayor gástrica. El examen intraoperatorio reveló una masa sólidoquística que surgía del mesocolon transverso. La cirugía consistió en la esplenectomía y exéresis en bloque del tumor. La paciente evolucionó de forma satisfactoria, dándose de alta al quinto día del postoperatorio. El diagnóstico de quiste epitelial esplénicose estableció en base al examen patológico de la pieza quirúrgica. Cursando su $6^{\circ}$ mes postoperatorio sin inconvenientes. Se realizó control tomográfico, que permitió verificar un bazo supernumerario funcionante.

PALABRAS CLAVE: Enfermedades del bazo; Quiste esplénico; Quiste epidérmico del bazo.

\section{INTRODUCCIÓN}

Los quistes esplénicos se dividen dependiendo del agente etiológico, en parasitarios y no parasitarios; diagnosticándose estos últimos preferentemente en áreas endémicas y son causados principalmente por infección por Echinococcus granulosus (Manterola et al., 2003; Ingle et al., 2014).

Por su parte, quistes primarios del bazo (QPB) o no parasitarios; son inusuales y, a menudo, un hallazgo incidental en la práctica quirúrgica. Es así como se ha reportado una prevalencia menor a 0,1 (Ingle et al., 2014).

Según la literatura existente, desde que Andral informó el primer caso en 1929, la clasificación de estas lesiones ha evolucionado hacia el sistema actual (Ingle et al., 2013, 2014); que los sistematiza según la presencia o ausencia de un revestimiento epitelial, etiología, patogénesis, etc. De este modo, se puede hablar de quistes tipo 1 , que son quistes verdaderos que tienen epitelio de revestimiento; y quistes tipo 2, que son quistes falsos sin revestimiento epitelial (Garg et al., 2013).
Los QPB, suelen ser asintomáticos y se diagnostican como hallazgo incidental en niños y adolescentes (Macheras et al., 2005; Garg et al.; Tajik et al., 2019). Su importancia clínica se asocia a su potencial de ruptura, infección y hemorragia (Tajik et al.).

El tratamiento de elección es la esplenectomía, ya sea total, parcial; vía abierta o laparoscópica (Chung et al., 2020).

La evaluación histopatológica y la inmunohistoquímica son la base para confirmar el subtipo de QPB y descartar una transformación maligna del epitelio pluripotencial (Manoj et al., 2012).

El objetivo de este manuscrito fue reportar un caso de QPB intervenido quirúrgicamente y revisar la evidencia existente respecto de sus características morfológicas, terapéuticas y pronósticas.

\footnotetext{
${ }^{1}$ Departamento de Cirugía y Centro de Estudios Morfológicos y Quirúrgicos (CEMyQ), Universidad de La Frontera, Chile.

${ }^{2}$ Clínica RedSalud Mayor, Temuco, Chile.
} 


\section{CASO CLÍNICO}

Este reporte, fue escrito siguiendo la guía Case Report Guidelines (CARE) (Gagnier et al., 2013).

Paciente de 18 años, sexo femenino, sin antecedentes mórbidos; que consultó en el Centro Médico de la clínica Red Salud Mayor de Temuco, Chile; dolor abdominal de tres semanas de evolución, asociada a sensación de bulto en el cuadrante superior del abdomen. A la exploración física, destacaba la palpación de una masa tumoral de gran tamaño, en el cuadrante superior izquierdo del abdomen.

Las pruebas de laboratorio con las que consultó se detallan en la Tabla I; pero de ellas, destaca una trombocitosis de $419.00010^{\wedge} 3 / \mathrm{ul}$, incremento de la VHS $(31 \mathrm{~mm} / \mathrm{h})$, cifras elevadas de Ca 125 (77U/mL), y del Ca 19-9 (133 U/mL).

Tabla I. Pruebas de Laboratorio Preoperatorias.

\begin{tabular}{lccc}
\hline \multicolumn{1}{c}{ Variables } & $\begin{array}{c}\text { Resultados } \\
\text { preoperatorios }\end{array}$ & $\begin{array}{c}\text { Resultados } \\
\text { postoperatorios * }\end{array}$ & $\begin{array}{c}\text { Valores } \\
\text { normales }\end{array}$ \\
\hline Hemoglobina (g/dL) & 12,8 & 12,1 & $12-16$ \\
Hematocrito $(\%)$ & 38,0 & 37,5 & $37-47$ \\
Leucocitos $\left(10^{\wedge} 3 / \mathrm{ul}\right)$ & 6,30 & 6,50 & $4-10$ \\
Plaquetas $\left(10^{\wedge} 3 / \mathrm{ul}\right)$ & 419 & 380 & $150-400$ \\
VHS $(\mathrm{mm} / \mathrm{hr})$ & 31,0 & 19,0 & $1-20$ \\
Creatinina (mg/dL) & 0,6 & 0,7 & $0,5-1,0$ \\
Glicemia (mg/dL) & 86 & 88 & $74-99$ \\
Proteínas totales $(\mathrm{g} / \mathrm{dL})$ & 7,5 & 7,4 & $6,4-8,3$ \\
Albúmina (g/dL) & 3,9 & 3,7 & $3,4-4,8$ \\
Protrombina (\%) & 71,1 & 75,0 & $70-100$ \\
PCR $(\mathrm{mg} / \mathrm{dL})$ & 14,3 & 9,4 & $0-10$ \\
LDH $(\mathrm{U} / \mathrm{L})$ & 200 & 185 & $143-279$ \\
AFP $(\mathrm{Ui} / \mathrm{mL})$ & 0,9 & 0,9 & $0-7,5$ \\
CEA $(\mathrm{ng} / \mathrm{mL})$ & $<0,5$ & $<0,5$ & $0-3$ \\
Ca 125 (U/mL) & 77,0 & 24,0 & $<35$ \\
Ca 19-9 $(\mathrm{U} / \mathrm{mL})$ & 133,0 & 28,8 & $<37$ \\
\hline
\end{tabular}

*: Resultados medidos al segundo mes postoperatorio. PCR:Proteína C reactiva.LDH: Lactato deshidrogenasa. AFP: Alfa fetoproteína. CEA:Antígeno carcinoembrionario.

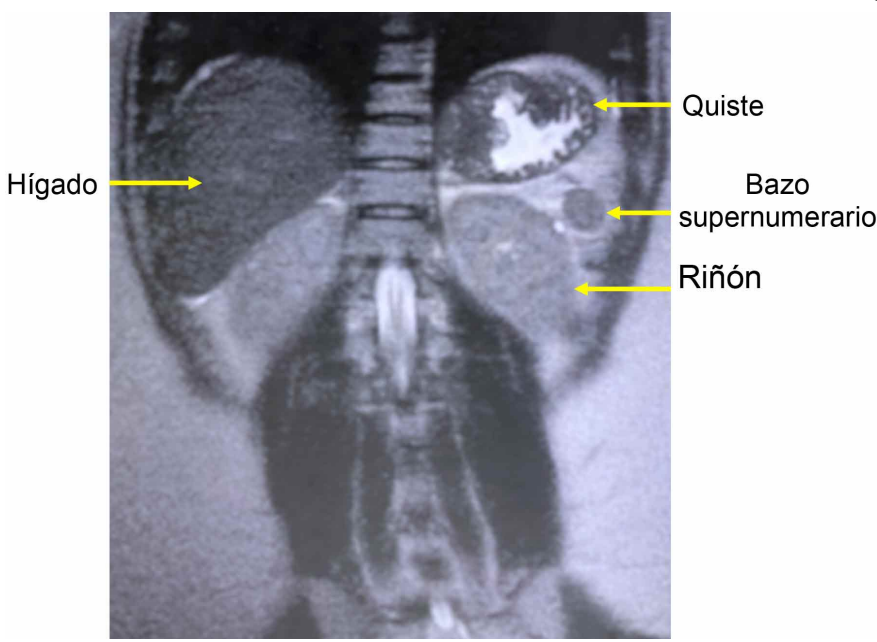

Fig. 1. TAC de abdomen: Reconstrucción coronal, en la que se aprecia imagen de lesión quística esplénica y bazo supernumerario.
Al momento de la consulta, expuso las imágenes y respectivos informes de una ecotomografía abdominal y una tomografía computarizada del abdomen (TAC). La primera sugería la existencia de una lesión quística en relación con el bazo y una hernia umbilical. En la TAC, se apreciaba ascitis multicompartimental, con protrusión umbilical, cambios inflamatorios del omento mayor, engrosamiento parietal y una lesión esplénica de aspecto quístico, de $25 \mathrm{~cm}$ de diámetro, sugerente de hidatidosis complicada (Fig. 1).

El desafío diagnóstico se relacionaba fundamentalmente con la etiología de la lesión y por ende con su pronóstico. Es así como en lo sindromático, se planteó como opción, un quiste hidatídico del bazo. Con esta orientación, se planificó una laparotomía exploradora.

Fue intervenida quirúrgicamente el 23 de junio de 2020, oportunidad en la que se verificó la existencia de ascitis a tensión, en cantidad de $1800 \mathrm{cc}$. Adherencias laxas y fibrosas del omento mayor a una gran masa quística que hacía cuerpo con el bazo, de aprox. $30 \mathrm{~cm}$ de diámetro mayor (Fig. 2). Bazo accesorio de $2 \mathrm{~cm}$ de diámetro mayor, localizado en la cola del páncreas (Fig. 3); y un defecto herniario umbilical, de aproximadamente $2 \mathrm{~cm}$ de diámetro.

Se realizó una laparotomía media supraumbilical, con ampliación en "J" a izquierda; aspiración de la ascitis antes descrita y liberación instrumental de adherencias. Luego, se movilizó el bazo, seccionando el ligamento esplenocólico, los vasos cortos; y ligando y seccionando por separado, la arteria y vena esplénica, con lino-000.

A continuación, se procedió a la reparación del defecto umbilical y al cierre de la pared abdominal, utilizando PDS1 continuo peritoneo-aponeurótico, vycril-00 al celular subcutáneo y grapas a la piel.

La evolución postoperatoria inmediata fue favorable, verificándose estabilidad hemodinámica, normoglicemia, diuresis normal; y tránsito intestinal gaseoso y de deposiciones a partir del segundo día del postoperatorio. Con pruebas de laboratorio dentro de límites normales, fue dada de alta al cuarto día postoperatorio.

El informe anatomopatológico de la pieza quirúrgica fue: "quiste epitelial esplénico roto, con signos de hemorragia reciente, negativo para neoplasia" (Fig. 4). Al estudio histológico, destacó la presencia de epitelio de revestimiento escamoso y cúbico (Fig. 5). El tamaño tumoral de la lesión fijada fue $20 \mathrm{~cm}$.

Al primer mes postoperatorio se constató una paciente en buenas condiciones generales, asintomática. 


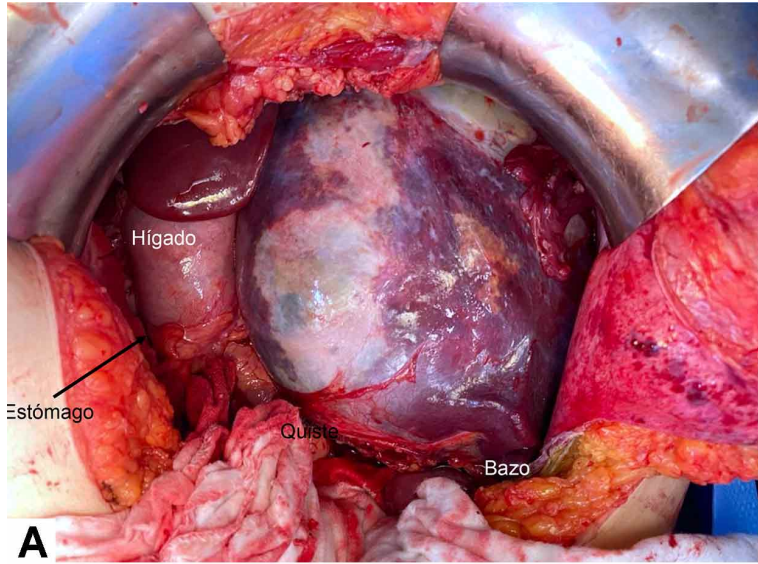

Fig. 2. A. Imagen intraoperatoria en la que se observa el quiste y sus relaciones con el bazo, estómago e hígado. B. Pieza quirúrgica en fresco. Se puede observar la relación quiste-bazo.
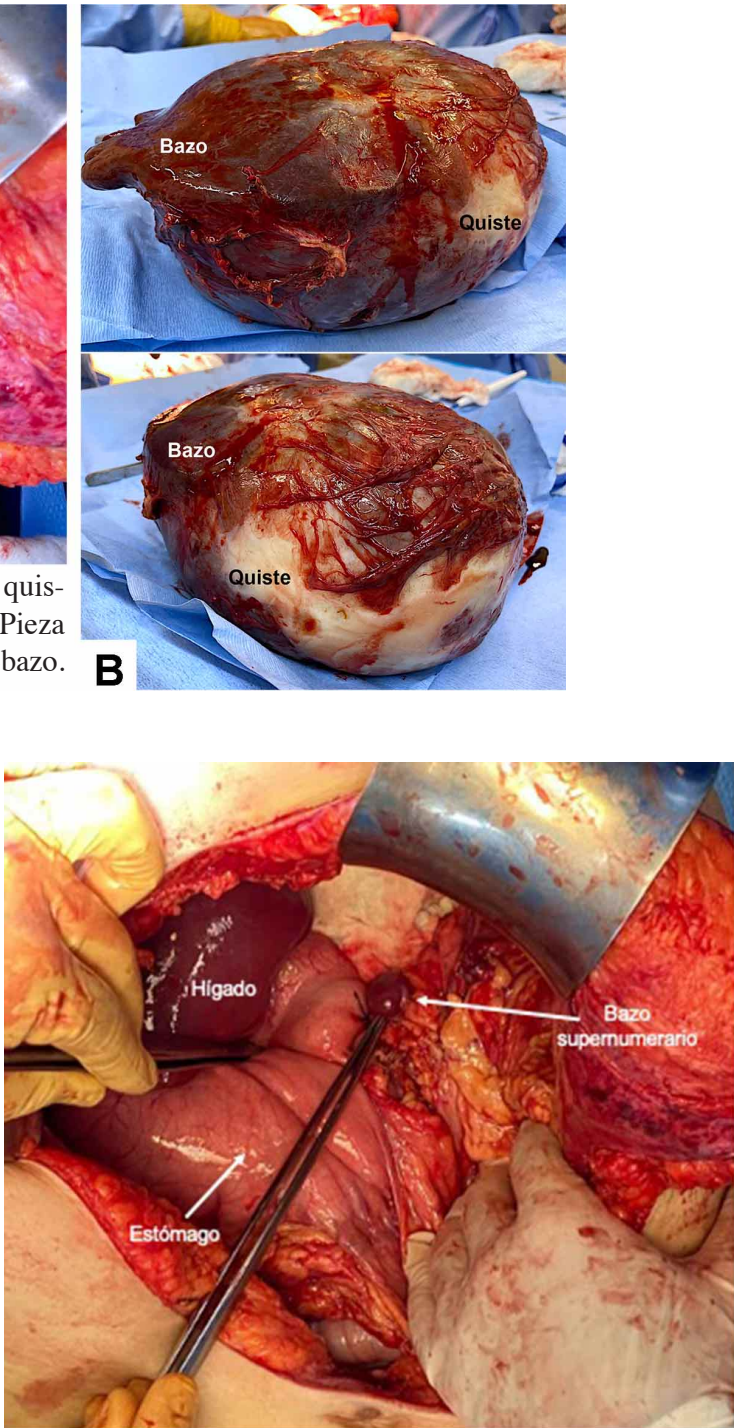

Fig. 3. Imagen intraoperatoria, en la que destaca la existencia de un bazo supernumerario, que fue conservado durante la cirugía.
Fig. 4. Pieza quirúrgica fijada.
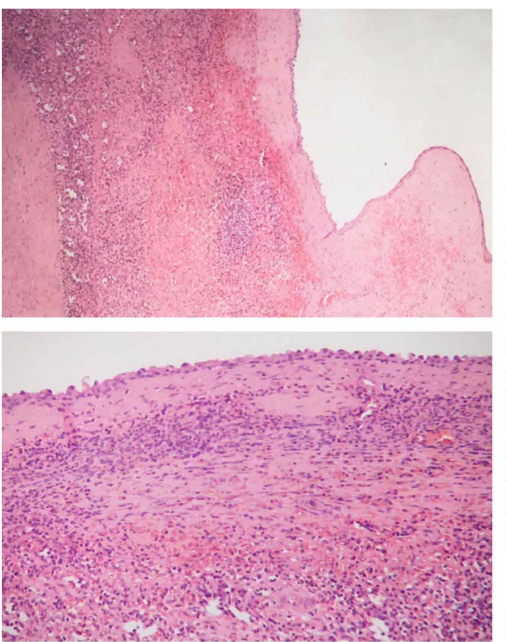
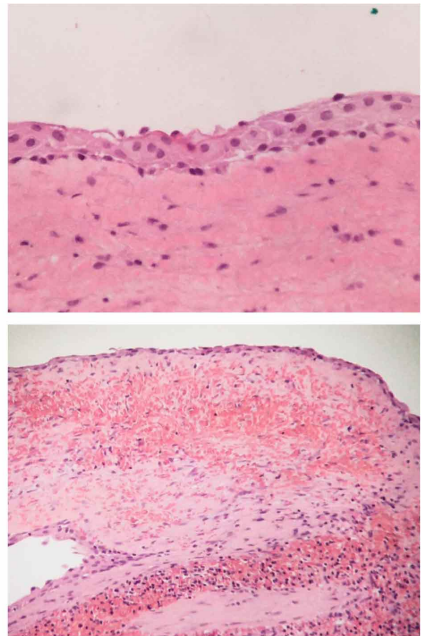

Fig. 5. Cortes histológicos. Se aprecia la presencia de epitelio de revestimiento escamoso y cúbico. 


\section{DISCUSIÓN}

Novedad de la propuesta: Se presenta un caso poco común de quiste epitelial esplénico, de gran tamaño, roto y con hemorragia, que fue extirpado quirúrgicamente de forma completa, con un curso postoperatorio adecuado.

Desde el punto de vista clínico, estas lesiones se caracterizan por un curso silencioso, y en la consulta inicial se puede evidenciar una masa palpable sensible (Sarmast et al., 2012; Álvarez et al., 2015; Z yluk \& Puchalski, 2016).

Existe evidencia en forma de reporte de casos, de pacientes con quistes epidermoides del bazo, que cursaron con incremento de CA 19-9, CA 125 y antígeno carcinoembrionario; así como de su normalización postoperatoria (Takagi et al., 2014; Buda et al., 2018; Okuno et al., 2019).

Las imágenes de elección para el estudio de estas lesiones son la TC y la RNM, ya que permiten verificar la relación del quiste con tejidos adyacentes (Pouché et al., 1999; Sarmast et al.).

La rotura de un quiste esplénico es una complicación rara, pero riesgosa. Por ello, tiene indicación quirúrgica perentoria (Res et al., 2019).

El tratamiento de elección de los quistes esplénicos, es la cirugía (Pouché et al.); ya sea la esplenectomía con remoción total de quiste por laparotomía o vía laparoscópica (Mertens et al., 2007; Z yluk \& Puchalski; Lobascio et al., 2017; Okuno et al.; Res et al.); como también la extirpación del quiste con conservación de bazo (Kapp et al., 2016). También existe evidencia reciente de exéresis utilizando robot (Kirih et al., 2020).

Limitaciones:Se ha de considerar que se trata de un caso único, y que la evidencia existente respecto de estas lesiones es escasa, como para poder comparar resultados.

\section{CONCLUSIONES}

Es difícil plantear una conclusión cuando se trata del reporte de un caso aislado y de una patología poco frecuente. Por ello, más que plantear una conclusión, comentar la lección aprendida respecto de la relevancia del diagnóstico precoz y la toma de decisiones oportunas que al respecto se han de considerar.
MANTEROLA, C. Epithelial splenic cyst. Morphological characteristics and postoperative evolution. Case report. Int. J. Morphol., 39(2):667-671, 2020.

SUMMARY: Primary splenic cysts (PSC) are rare lesions in surgical pathology; those symptomatic, or greater than $5 \mathrm{~cm}$, should be treated surgically to avoid the risk of complications. A histopathological examination should be performed to confirm the splenic cyst subtype and rule out a possible malignant transformation of the pluripotential epithelial lining. The aim of this manuscript was to report a case of PSC who had undergone surgery and to review the existing evidence regarding its morphological, therapeutic and prognostic characteristics. An 18year-old woman (MAC), consulted for progressive abdominal distention of several months of evolution. Abdominal tomography revealed the existence of a large heterogeneous mass, $21 \mathrm{~cm}$ in diameter, in relation to the transverse colon and the greater gastric curve. Intraoperative examination revealed a solid cystic mass arising from the transverse mesocolon. Surgery consisted of splenectomy and in-block excision of the tumor. The patient evolved satisfactorily and was discharged on the fifth postoperative day. Diagnosis of epithelial splenic cyst was established based on the pathological examination of the surgical specimen. At six months postoperative the patient had evolved satisfactorily without complications. Following abdominal tomography control a functioning supernumerary spleen was confirmed.

KEY WORDS: Splenic Diseases; Splenic cyst; Splenic epidermal cyst; Epithelial cyst of spleen.

\section{REFERENCIAS BIBLIOGRÁFICAS}

Álvarez, A.; Mir, V.; Tort, J.; Sala, J. \& Culubret, M. Primary splenic cyst. Semergen, 41(8):e81-3, 2015

Buda, N.; Wszo?ek, A.; S’ledzin'ski, M.; Z awrocki, A. \& Sworczak, K. Epidermoid splenic cyst with elevated serum level of CA19-9. Korean J. Intern. Med., 33(5):1032-3, 2018.

Chung, P.; Swinson, B.; O'Rourke, N. \& Schmidt, B. Massive splenic cyst in pregnancy: case report. BMC Pregnancy Childbirth, 20:273, 2020.

Gagnier, J. J.; Riley, D.; Altman, D. G.; Moher, D.; Sox, H.; Kienle, G. \& CARE Group. The CARE guidelines: consensus-based clinical case reporting guideline development. Dtsch. Arztebl. Int., 110(37):603-8, 2013.

Garg, M.; Kataria, S. P.; Sethi, D. \& Mathur, S. K. Epidermoid cyst of spleen mimicking splenic lymphangioma. Adv. Biomed. Res., 2:49, 2013.

Ingle, S. B.; Hinge Ingle, C. R. H. \& Patrike, S. Epithelial cysts of the spleen: a minireview. World J. Gastroenterol., 20(38):13899-903, 2014.

Ingle, S. B.; Hinge, C. R. \& Jatal, S. N. An interesting case of primary epithelial cyst of spleen. Indian J. Pathol. Microbiol., 56(2):181-2, 2013.

Kapp, J.; Lewis, T.; Glasgow, S.; Khalil, A. \& Anjum, A. Spleen preserving management of a non-parasitic splenic cyst in pregnancy. Ann.R. Coll. Surg. Engl., 98(7):e114-7, 2016.

Kirih, M. A.; Liang, X.; Xie, Y.; Cai, J.; Zheng, J.; Xu, F.; He, S.; Tao, L. \& Ali Abdi, F. Robot-assisted partial splenectomy for splenic epidermoid cyst. Case Rep. Surg., 2020:6245909, 2020.

Lobascio, P.; Carbotta, G.; Laforgia, R.; Fedele, S.; Sederino, M. G.; Minafra, M.; Delvecchio, A.; Ferrarese, F. \& Palasciano, N. Total laparoscopic splenectomy for giant epidermoid cyst: a case report. $G$. Chir., 38(4):202-4, 2017. 
Macheras, A.; Misiakos, E. P.; Liakakos, T.; Mpistarakis, D.; Fotiadis, C. $\&$ Karatzas, G. Non-parasitic splenic cysts: a report of three cases. World J. Gastroenterol., 11(43):6884-7, 2005.

Manoj, M. G.; Misra, P. \& Kakkar, S. Multilocular epithelial cyst of spleen: a rare occurrence. Indian J. Pathol. Microbiol., 55(4):602-4, 2012.

Manterola, C.; Vial, M.; Losada, H.; Fonseca, F.; Bustos, L.; Muñoz, S. \& Barroso, M. Uncommon locations of abdominal hydatid disease. Trop. Doct., 33(3):179-80, 2003.

Mertens J.; Penninckx, F.; DeWever, I. \& Topal, B. Long-term outcome after surgical treatment of nonparasitic splenic cysts. Surg. Endosc., 21(2):206-8, 2007.

Okuno, M.; Yuasa, N.; Takeuchi, E.; Goto, Y.; Miyake, H.; Nagai, H.; Yoshioka, Y. \& Miyata, K. A case of ruptured splenic cyst with elevated serum levels of CEA treated by laparoscopic unroofing. Clin. J. Gastroenterol., 12(6):642-9, 2019.

Pouché, A.; Biasca, F.; Laffranchini, G.; Lanzi, S.; Giampaoli, F. \& Coniglio, A. Benign non-parasitic cysts of the spleen. Ann. Ital. Chir., 70(1):838,1999

Res, L. C. S.; Knook, M. T. T.; Hazelbag, H. M. \& Guicherit, O. R. Spontaneous rupture of a non-parasitic splenic cyst. BMJ Case Rep., 12(10):e231473, 2019.

Sarmast, A. H.; Showkat, H. I.; Parray, F. Q. \& Lone, R. Non parasitic splenic cyst: a case report. Acta Med. Iran., 50(12):849-51, 2012.

Tajik, P.; Goudarzian, A. H. \& Pourzahabi, Z. A 14-year girl with abdominal pain: case report of splenic cyst. Gastroenterol. Hepatol. Bed Bench, 12(3):260-2, 2019.

Takagi, K.; Takayama, T.; Moriguchi, M.; Hasegawa, H.; Niide, O.; Kanamori, N.; Higaki, T. \& Sugitani, M. Gastrointestinal: case of accidentally discovered splenic epidermoid cyst with serum CA19-9 elevation. J. Gastroenterol. Hepatol., 29(2):231, 2014.

Z yluk, A. \& Puchalski, P. Giant splenic cyst: a case report. Pomeranian J. Life Sci., 62(4):64-6, 2016.

\author{
Dirección para correspondencia: \\ Dr. Carlos Manterola \\ Departamento de Cirugía y CEMyQ \\ Universidad de La Frontera \\ Temuco \\ CHILE
}

\section{E-mail: carlos.manterola@ufrontera.cl}

Recibido : 16-12-2020

Aceptado : 28-01-2021 